\title{
A missense mutation within the fork-head domain of the Forkhead box G1 (FOXG1) gene affects its nuclear localization
}

\begin{tabular}{|c|c|}
\hline Journal: & Human Mutation \\
\hline Manuscript ID: & humu-2010-0315.R2 \\
\hline Wiley - Manuscript type: & Mutation in Brief \\
\hline $\begin{array}{r}\text { Date Submitted by the } \\
\text { Author: }\end{array}$ & $16-N o v-2010$ \\
\hline Complete List of Authors: & $\begin{array}{l}\text { Le Guen, Tangui; Université Paris Descartes, Institut Cochin, } \\
\text { Inserm U1016, UMR8104, Institut Cochin } \\
\text { Fichou, Yann; Université Paris Descartes, Institut Cochin, Inserm } \\
\text { U1016, UMR8104, Institut Cochin } \\
\text { Nectoux, Juliette; Université Paris Descartes, Institut Cochin, } \\
\text { Inserm U1016, UMR8104, Institut Cochin } \\
\text { Bahi-Buisson, Nadia; Hôpital Necker-Enfants Malades, Assistance } \\
\text { Publique Hôpitaux de Paris, Service de Neuropédiatrie } \\
\text { Rivier, Francois; Hopital Gui de Gaillac, CHU Montpellier, Pediatric } \\
\text { Neurology } \\
\text { Boddaert, Nathalie; Hopital Necker-Enfants Malades, Assistance } \\
\text { Publique Hôpitaux de Paris, Pediatric Radiology } \\
\text { Diebold, Bertrand; AP-HP, Hôpital Cochin, Laboratoire de Biochimie } \\
\text { et Génétique Moléculaire } \\
\text { Heron, Delphine; Hôpital Pitié Salpétrière, Assistance Publique } \\
\text { Hôpitaux de Paris, Service de Génétique Médicale } \\
\text { Chelly, Jamel; Université Paris Descartes, Institut Cochin, Inserm } \\
\text { U1016, UMR8104, Institut Cochin } \\
\text { Bienvenu, Thierry; Inserm, Université Paris Descartes, Institut } \\
\text { Cochin, CNRS (UMR8104) }\end{array}$ \\
\hline Key Words: & Rett syndrome, FOXG1, Microcephaly, Nuclear speackles \\
\hline
\end{tabular}




\title{
A missense mutation within the fork-head domain of the Forkhead box G1 (FOXG1) gene affects its nuclear localization

\author{
Tangui Le Guen 1,2, Yann Fichou 1,2, Juliette Nectoux 1,2, Nadia Bahi-Buisson 2,3, \\ François Rivier ${ }^{4}$, Nathalie Boddaert ${ }^{5}$, Bertrand Diebold ${ }^{6}$, Delphine Héron ${ }^{7}$, Jamel Chelly ${ }^{1,2}$, Thierry Bienvenu ${ }^{1,2}$ \\ 1. Inserm, U1016, Paris, France. \\ 2. Institut Cochin, Université Paris Descartes, CNRS (UMR 8104), Paris, France. \\ 3. Pediatric Neurology, Necker-Enfants Malades Hospital, AP-HP, Paris, France. \\ 4. Pediatric Neurology, Gui de Gaillac Hospital, Montpellier University Hospital, Montpellier, France \\ 5. Pediatric Radiology, Necker Enfants Malades Hospital, AP-HP, Université Paris Descartes, Paris, France \\ 6. Laboratoire de Biochimie et Génétique Moléculaire, AP-HP, Cochin Hospital, Paris, France \\ 7. Service de Génétique Médicale, Pitié-Salpêtrière Hospital, Paris, France.
}

${ }^{*}$ Correspondence to Dr. Thierry Bienvenu, Université Paris Descartes, Institut Cochin, 24 rue du Faubourg St Jacques 75014 Paris, France, Phone : 33 +1 444124 79, Fax : 33 + 5841 15 80, Email : thierry.bienvenu @ inserm.fr

Contract grant sponsor: Institut National de la Santé et de Recherche Médicale (ANR-08-e-rare EuroRETT Network)

Short Title: Missense mutation within the fork-head domain of the FOXG1 gene

Communicated by <Please don't enter $>$

ABSTRACT: The forkhead box G1 (FOXG1) gene has recently been associated with the congenital variant of Rett syndrome, and so far 17 mutations have been reported. We screened the coding region in 150 patients affected by postnatal microcephaly, and identified two mutations: the c.326C>T (p.P109L) substitution inherited from the healthy father; and the de novo c.730C $>T$ transition, which induces the p.R244C mutation within the DNA-binding forkhead domain. This latter mutation is carried by an 8-year-old girl, who presented a phenotype reminiscent of the congenital variant of Rett syndrome. Immunofluorescence analysis of the wild-type protein revealed a

Received <date>; accepted revised manuscript $<$ date $>$.

\section{(C) 2010 WILEY-LISS, INC.}




\section{$2<$ First Author et al.>}

homogeneous nuclear staining excluding the nucleoli, while the p.R244C mutant showed abnormal nuclear foci in a large proportion of cells, suggesting that its mislocalization may reduce and/or impair target recognition. Interestingly, this missense mutation results in a mislocalization of FoxG1 to specific nuclear foci referred to as nuclear speckles, and affects the cyclin-dependent kinase inhibitor p21 CDKN1A expression. Because CDKL5, which is involved in the early-onset variant of Rett syndrome, is also located in these speckles, we suggest that disregulation of the dynamic behaviour of nuclear speckles may functionally link these two proteins, which are both involved in atypical forms of Rett syndrome.@2010 Wiley-Liss, Inc.

KEY WORDS: FOXG1; Rett syndrome; Microcephaly; Encephalopathy; Nuclear speckles

\section{INTRODUCTION}

The mammalian forkhead family belongs to the large family of over 100 known forkhead genes in animals [Solomon et al., 2003a]. All forkhead family members contain the $\sim 100$ amino acid, monomeric DNA "wingedhelix" binding domain of the founding members: Drosophila forkhead [Lee and Frasch, 2004] and hepatocyte nuclear factor 3a [Tao and Lai, 1992]. Forkhead gene products play pivotal roles in organogenesis, including patterning and morphogenesis, through the regulation of the proliferation and cell fate specification [Solomon et al., $\underline{2003 \mathrm{~b}}]$.

The neocortex develops from the progenitor cells of the rostral neural plate, the telencephalic neuroepithelial cells. Following a period of uniform proliferation, cerebral cortical progenitors generate neurons asynchronously beginning at embryonic day 11 (E11) in mouse. Progenitors undergo asymmetric cell divisions in which one daughter cell differentiates while the other divides. The proportion of cells with asymmetric divisions steadily increases over a period of several days. At the end of the neurogenetic period (E17), both daughter cells differentiate, resulting in the depletion of the progenitor pool. The duration of the neurogenetic period is a critical determinant driving the quantity of neurons generated within the cerebral cortex. Three factors have a major influence on the cellular output from the proliferative zones: the rate of cell proliferation, the rate of cell differentiation, and the rate of cell death. The progenitor cells of the telencephalon are identifiable as early as the eight-somite stage (E8.5) by the expression of one of the members of the forkhead box family, the FoxG1 transcription factor, also known as the winged-helix (WH) protein brain factor (BF-1) [Murphy et al., 1994].

FoxG1 (MIM 164874) is a DNA-binding transcription factor with a forkhead binding domain (FBD; amino acids (AA) 181-275), which represses target genes during brain development by recruiting transcriptional corepressor proteins via two protein-binding domains: the Groucho-binding domain (GBD; AA 307-317) and the JARID-1B binding domain (JBD; AA 383-406).

In a recent series of articles, several groups identified ten different mutations in FOXGl as the cause of the congenital form of Rett syndrome (RTT, OMIM 312750) [Ariani et al., 2008; Philippe et al., 2010; Mencarelli et al., 2010; Bahi-Buisson et al., 2010]. All patients fulfilled the criteria of the congenital variant of RTT except one presenting a classical form of Rett syndrome [Philippe et al., 2010]. The congenital variant of RTT, which was initially described by Rolando in 1985 [Rolando et al., 1985], involves girls that present with a normal perinatal 
period followed by a phase of developmental regression at the age of 3-6 months. At birth, head circumference is normal while a deceleration of growth is observed soon afterwards, resulting in a severe microcephaly. Patients with FOXG1 mutations usually exhibit a phenotype reminiscent of the congenital RTT variant. Motor development is severely impaired and voluntary hand-use is absent. In contrast with classic RTT, patients show poor eye contact. Typical stereotypic hand movements with hand-washing and hand-mouthing activities are observed continuously. Several patients show abnormal tongue movements, as well as jerky movements of the limbs. Brain MRI typically shows corpus callosum hypoplasia, abnormal ventricles, and abnormal white matter in most cases, while epilepsy is a variable sign [Bahi-Buisson et al., 2010]. Scoliosis and autonomic neurovegetative symptoms typical of Rett syndrome are frequently present [Philippe et al., 2010].

To date, only a few missense mutations have been reported in the FOXG1 gene. In this report, the gene was screened by direct sequencing in more than 150 sporadic cases of girls with microcephaly, stereotypic hand movements and jerky movements. A novel missense mutation within the DNA-binding domain was identified and subsequent studies by genetic and cellular approaches showed that the nuclear localization of the protein is impaired. These results provide new insights into the understanding of FoxG1 dysfunction and its associated phenotype.

\section{MATERIALS AND METHODS}

\section{Patients}

As part of the Rettsearch program (SYRENE program), 150 female patients with a clinical diagnosis of severe congenital encephalopathy with microcephaly were selected for the study. The cohort included nine females with the classical form of Rett syndrome, 19 patients with the early seizure onset variant, 7 patients with the congenital variant of Rett syndrome, and 115 patients showing some of the features observed in Rett syndrome. This last heterogeneous group of patients included patients with mental retardation, autistic features, stereotypic hand movements, progressive microcephaly, epileptic encephalopathy, and/or severe congenital encephalopathy without recognizable aetiology. In all selected individuals, neither point mutation nor large rearrangement in the $M E C P 2$ and $C D K L 5$ genes had been identified. Methylation studies for Angelman syndrome and chromosome analysis were also normal.

\section{DNA mutation analysis}

DNA was extracted from peripheral blood using standard methods. All blood samples were obtained after provision of informed consent. DNA samples were screened for mutations in FOXG1 (GenBank reference NG_009367.1)) using PCR amplification and direct sequencing. Primer sequences and PCR conditions are available upon request to the corresponding author. Sequencing reactions were carried out with the BigDye Terminator v1.1 Cycle Sequencing Kit (Applied Biosystems, Courtaboeuf, France) and loaded on the ABI 3100 Genetic Analyzer (Applied Biosystems). Nucleotide numbering reflects cDNA numbering with +1 corresponding to the A of the ATG initiation codon (codon 1) in the reference sequence, according to journal guidelines (www.hgvs.org/mutnomen). Mutation nomenclature follows the recommended guidelines of the human genome 


\section{$4<$ First Author et al.>}

variation society (www.HGVS.org). Nucleotide numbering is based on cDNA reference sequences GenBank accession number NM_005249.3. Detection of large rearrangements of the FOXG1 gene was assessed by quantitative, real-time PCR carried out with the SYBR Green PCR Master Mix (Applied Biosystems) on an ABI Prism 7000 Sequence Detection System (Applied Biosystems) according to the manufacturer's instructions. Primers were designed with Primer Express software and are available upon request to the corresponding author. After PCR amplification, the purity of products was determined by a melting-curve analysis, and all samples indicating a single amplification product were considered positive for further analysis. The identity and size of the single PCR product were also confirmed by agarose gel electrophoresis. For each sample, a relative quantification of the copy number of the FOXG1 gene was calculated by the $\triangle \mathrm{Ct}$ method as described by the manufacturer using the $A L B$ gene as the calibrator for normalization.

\section{Plasmid construction and production}

The human FOXG1 open-reading frame was extracted from the BC035020 clone (cDNA clone IMAGE: 4823883, Thermo Scientific Open Biosystems, Huntsville, AL, USA) by PCR amplification with forward (F) and reverse (R) primers flanked by BglII and BamHI restriction sites, respectively: 5'-

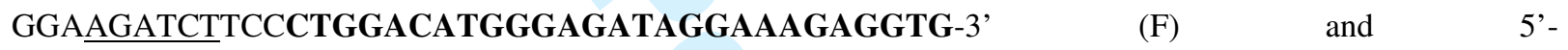
CGCGGATCCGCGATGTATTAAAGGGTTGGAAGAAGACCCCTG-3' (R) (restriction sites are underlined; FOXG1 sequences are in bold). This amplification removes the initiation codon ATG from the sequence and allows its subcloning as the C-terminal part of a fusion protein. The PCR was carried out with the PCRx Enhancer System (Invitrogen, Cergy-Pontoise, France) with $10 \mathrm{ng}$ of template plasmid DNA, 1X PCRx Amplification Buffer, 2X PCRx Enhancer Solution, $2 \mathrm{mM} \mathrm{MgSO}_{4}, 250 \mu \mathrm{M}$ dNTP each, $0.5 \mu \mathrm{M}$ each primer, and the Platinum ${ }^{\circledR}$ Taq DNA Polymerase. Cycle conditions were: an initial denaturation step at $95^{\circ} \mathrm{C}$ for $2 \mathrm{~min}$; followed by 40 cycles of denaturation at $95^{\circ} \mathrm{C}$ for $45 \mathrm{sec}$, annealing at $62^{\circ} \mathrm{C}$ for $30 \mathrm{sec}$, extension at $68^{\circ} \mathrm{C}$ for $1 \mathrm{~min} 45 \mathrm{sec}$; and a final extension step at $68^{\circ} \mathrm{C}$ for $2 \mathrm{~min}$. PCR products were loaded on a $2 \%$ agarose gel and purified with the QIAquick Gel Extraction Kit (Qiagen, Courtaboeuf, France). The purified fragments were subsequently cloned within the pCR $4^{\circledR}-\mathrm{TOPO}^{\circledR}$ (Invitrogen) vector according to the protocol described by the manufacturer. The cloning product was transformed into XL1-Blue Supercompetent Cells (Stratagene, purchased from Agilent Technologies, Massy, France) cultured in a selective medium. The reported $\mathrm{G}>\mathrm{C}$ substitution at codon 82 , which converts a proline to an arginine, was found in the clone and reversed by directed mutagenesis using the QuikChange ${ }^{\circledR}$ Site-Directed Mutagenesis Kit (Stratagene) with oligonucleotides 5'-CCCCCGGCACCGCAGCCCCCC-3' (F) and 5'GGGGGGCTGCGGTGCCGGGGG-3' (R) (converted codon underlined; converted nucleotide in bold) according to the manufacturer's instructions.

To generate the expression vectors, the TOPO-TA cloning product containing the human FOXG1 sequence, as well as the pEGFP-C1 Vector (Clontech, purchased from Ozyme, Saint-Quentin-en-Yvelines, France) were digested with both the BgIII and BamHI restriction enzymes, and the linearized pEGFP-C1 Vector was treated with Alkaline Phosphatase (New England Biolabs, purchased from Ozyme, Saint-Quentin-en-Yvelines, France). Digestion products were loaded on a $0.8 \%$ agarose gel and extracted as described above. Insert and vector were 
then ligated with the T4 DNA Ligase (New England Biolabs) overnight at $4{ }^{\circ} \mathrm{C}$, and transformed into XL10-Gold ${ }^{\circledR}$ Ultracompetent Cells (Stratagene) cultured in a selective medium, to generate the pEGFP-FOXG1 recombinant plasmid producing an EGFP-FOXG1 fusion protein. After direct sequencing, this wild-type construct serves as a template to generate the p.R244C mutant (c.730C $>$ T) by directed mutagenesis with the QuikChange ${ }^{\circledR}$ XL SiteDirected Mutagenesis Kit (Stratagene) and the following oligonucleotides: 5'CGTGAAGGTGCCGTGCCACTACGACGA-3' (F) and 5'-TCGTCGTAGTGGCACGGCACCTTCACG-3' (R) (converted codon underlined; converted nucleotide in bold). Sequences of intermediate and final plasmid constructs were systematically controlled by direct sequencing.

\section{Cell culture, transfection and immunofluorescence}

The DAOY medulloblastoma cell line and the HeLa human cervix carcinoma cells (ATCC ${ }^{\circledR}$ Number: CCL$2^{\mathrm{TM}}$ ) were maintained in Dulbecco's modified Eagle's medium supplemented with $10 \%$ fetal calf serum and antibiotics at $37^{\circ} \mathrm{C}, 5 \% \mathrm{CO}_{2}$ under humidified atmosphere. One day before transfection, cells were seeded onto round coverslips and cultured in minimal medium. They were either mock-transfected or transfected with the plasmid encoding an EGFP-tagged fusion protein using Lipofectamine ${ }^{\mathrm{TM}} 2000$ (Invitrogen) as the transfection reagent following the manufacturer's instructions. Forty-eight hours after transfection, cells were fixed with methanol $100 \%,-20^{\circ} \mathrm{C}$ for 5 minutes. For direct fluorescence, coverslips were mounted with Vectashield ${ }^{\circledR}$ Mounting Medium with DAPI (Vector Laboratories, purchased from Abcys, Paris, France) and visualised with a Leica DMRA2 fluorescence microscope. All subsequent steps were carried out at room temperature unless indicated. For indirect immunofluorescence, cells were permeabilized with $0.2 \%$ Triton X-100, 15 min, and unspecific sites were blocked with a 5\% non-fat milk solution in PBS 1X, $30 \mathrm{~min}$. Cells were incubated with the mouse monoclonal anti-splicing factor SC-35 antibody (Sigma-Aldrich, dilution 1:200), overnight at $4^{\circ} \mathrm{C}$, and then with a Texas Red-conjugated secondary anti-mouse IgG (dilution 1:4000) for 1 hour. After extensive washing, coverslips were mounted as described above.

\section{Cell fractionation extracts and western blot analysis}

Cell fractionation to isolate the nuclear proteins from the cytosolic proteins was carried out as described before [Regad et al., 2007]. All fractions were suspended with Laemmli Sample Buffer 2X (BioRad, Richmond, CA, USA), and equivalent cell ratios (1:1) of nuclear $v s$ cytosolic proteins were loaded on a SDS-polyacrylamide gel to estimate the enrichment of the fusion proteins in either subcellular compartment. After gel electrophoresis, proteins were transferred on a Hybond-C nitrocellulose membrane (GE Healthcare), which was subsequently blocked with a TBS-Tween $200.05 \% / B S A 5 \%$ solution, and incubated overnight at $4{ }^{\circ} \mathrm{C}$ with a rabbit anti-GFP polyclonal antibody (Santa Cruz Biotechnology, Inc.; dilution 1:1000). The membrane was further extensively washed and incubated with a horseradish peroxidase (HRP)-conjugated anti-rabbit $\operatorname{IgG}$ (dilution 1:5000), and developed with the enhanced chemiluminescence method (ECL; GE Healthcare). The membrane was reprobed for $\beta$-actin (Santa Cruz Biotechnology, Inc.; dilution 1:10000) for fractionation checking. 


\section{$6<$ First Author et al.>}

\section{Cell culture, transfection, and RT-PCR analysis}

The human neuroblastoma SH-SY5Y cell line was cultured in 1:1 mixture of Dulbecco's modified Eagle's medium (DMEM) and Ham's F12 medium (Sigma) containing $100 \mathrm{U} / \mathrm{ml}$ penicillin, $100 \mathrm{mg} / \mathrm{ml}$ streptomycin and $10 \%$ fetal calf serum (FCS) and incubated at $37^{\circ} \mathrm{C}, 5 \% \mathrm{CO}_{2}$ in a humidified atmosphere. Five hundred thousand cells were seeded in $100 \mathrm{~mm}$ collagen-coated cell culture dishes. After 24 hours, the medium was replaced by a 1:1 mixture of DMEM and Ham's F12 medium with 0.5\% FCS and retinoic acid (RA; final concentration $5 \mu \mathrm{M}$ ) for differentiation. After five days in these conditions, cells were seeded in 6-well plates to reach 50-70\% confluency. Cells were then transfected with either FOXG1 constructs (see the cell culture, transfection and immunofluorescence section) using Lipofectamine 2000 (Invitrogen, CA) as the transfection reagent according to the manufacturer's instructions, and incubated for 4-6 hours. The transfection medium was then replaced by the complete growth medium. Cells were cultured for 48 hours, and (GFP+)-transfected cells were collected and selected using a FACScan flow cytometer. Total RNA was extracted from the sorted SH-SY5Y cells by Trizol Reagent (Invitrogen) according to the manufacturer's instructions, and converted to cDNA prior to quantitative, real-time PCR analysis carried out with the SYBR Green PCR Master Mix (Applied Biosystems) on an ABI Prism 7500 Sequence Detection System (Applied Biosystems) according to the manufacturer's instructions. CDKN1A expression was assessed by quantitative Reverse Transcriptase (RT)-PCR (primer sequences available upon request to the corresponding author). After PCR amplification, a dissociation protocol was performed to determine the melting curve of the PCR product. Reactions with melting curves indicating a single amplification product were considered positive for further analysis. The identity and expected size of the single PCR product were also confirmed by agarose gel electrophoresis. For each sample, a relative quantification of the expression of each individual gene of interest was calculated by the comparative $\Delta \Delta \mathrm{Ct}$ method as described by the manufacturer, using HPRT as the calibrator for normalization. Statistical analysis was carried out using the nonparametric Mann-Whitney test. A $p$-value $<0.05$ was considered as significant.

\section{RESULTS}

\section{Molecular analysis and case report}

The whole coding sequence of the FOXG1 gene was screened by direct sequencing in 150 clinically wellcharacterized girls with severe microcephaly. Two missense mutations were identified: the c.326C $>\mathrm{T}$ transition and the $c .730 \mathrm{C}>\mathrm{T}$ transition, which are predicted to result in p.P109L and the p.R244C changes at the amino acid level, respectively. The first variant was subsequently identified in her healthy father at the heterozygous state, suggesting that it is a non-pathogenic polymorphism.

Conversely, the c.730C $>\mathrm{T}$ transition is a de novo mutation (data reviewed but not shown) that was not detected in more than 100 control female individuals of different ethnic origins. The patient carrying this mutation is an 8-year-old girl, third child of non-consanguineous healthy parents. Prenatal ultrasound at 33 weeks of gestation showed a relative microcephaly $\left(10^{\text {th }}\right.$ percentile $)$ without cortical malformation. She was born full-term by spontaneous delivery, with normal neonatal parameters but relative small head circumference (OFC) $(33 \mathrm{~cm}$; 
$10^{\text {th }}$ percentile). On first examination at 4 months old, deceleration of head growth $\left(3^{\text {rd }}\right.$ percentile) with good eye contact and smile, moderate axial hypotonia and limb hypertonia were noted. First brain MRI performed at 7 months old showed frontally predominant pachygyria with thick frontal gyri and reduced white matter (Fig. 2 AD). Initially she was suspected to present with cortical malformation and was referred for anterior pachygyria. At 7 months old, she developed hyperkinetic and dyskinetic movement disorder but neither prehension, nor babbling. In addition, sleep disorders and intermittent unexplained crying were reported. She never presented myoclonia or seizures. EEG was normal, as well as fundoscopy. Progressively, she developed severe mental retardation although she made constant progress. Secondary deceleration of head growth leads to absolute microcephaly. At last evaluation at 8 years of age, microcephaly was severe $\left(45.5 \mathrm{~cm} ;<3^{\text {rd }}\right.$ percentile), she was able to walk with aid, presented hyperkinetic and choreic movements, and dyskinetic tongue movement and hand stereotypies. Hand stereotypies were variable, but hand-mouthing stereotypies were predominant. She also presented pyramidal signs with mild spasticity. Eye contact dramatically improved while verbal language did not. Brain MRI performed at 6 years old showed frontal gyral simplification without pachygyria without hypomyelination, and mildly reduced white matter (Fig. 2 E-H). Altogether, the girl presented a phenotype reminiscent of the congenital variant of Rett syndrome.

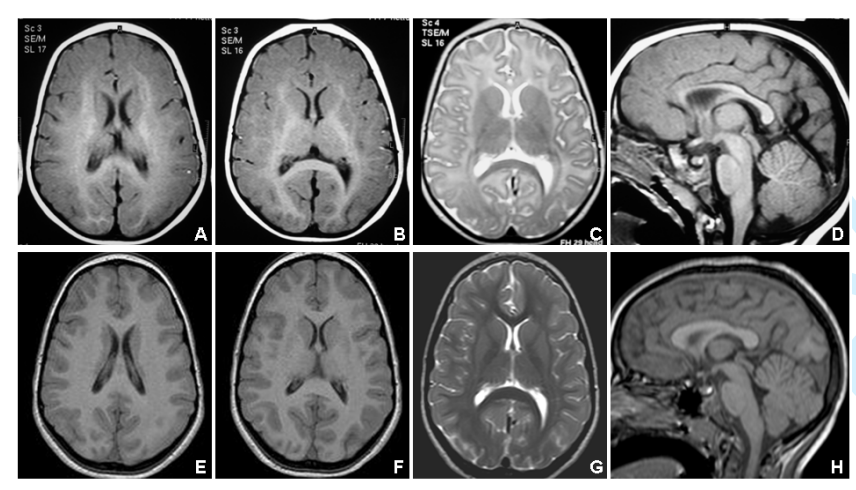

Figure 1. A-D Brain MRI at 7 months of age. T1 weighted sequences show frontal pachygyria with thick frontal gyri (A; B). On T2 weighted sequence, mild myelination delay with only internal capsule myelinated (C). T1 sagital section shows thin corpus callosum and normal fossa posterior (D). E-H refer to age-matched control.

The resulting change at the amino acid level is predicted to replace an arginine by a cysteine residue (p.R244C) in the highly evolutionarily conserved DNA-binding forkhead domain of the protein (Fig. 2), and may thus be critical for the function of the protein. 
A

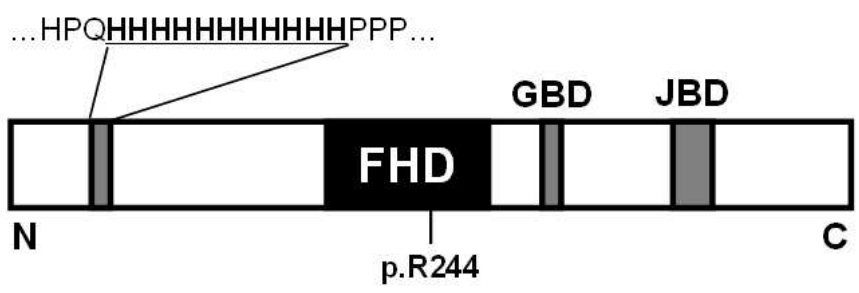

B

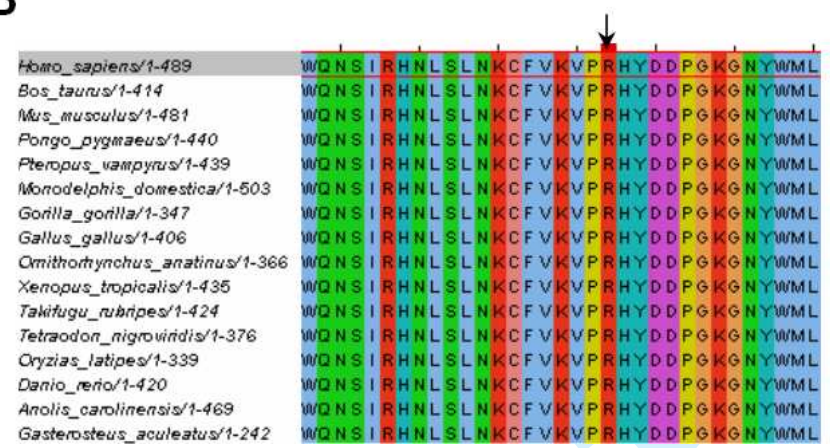

C

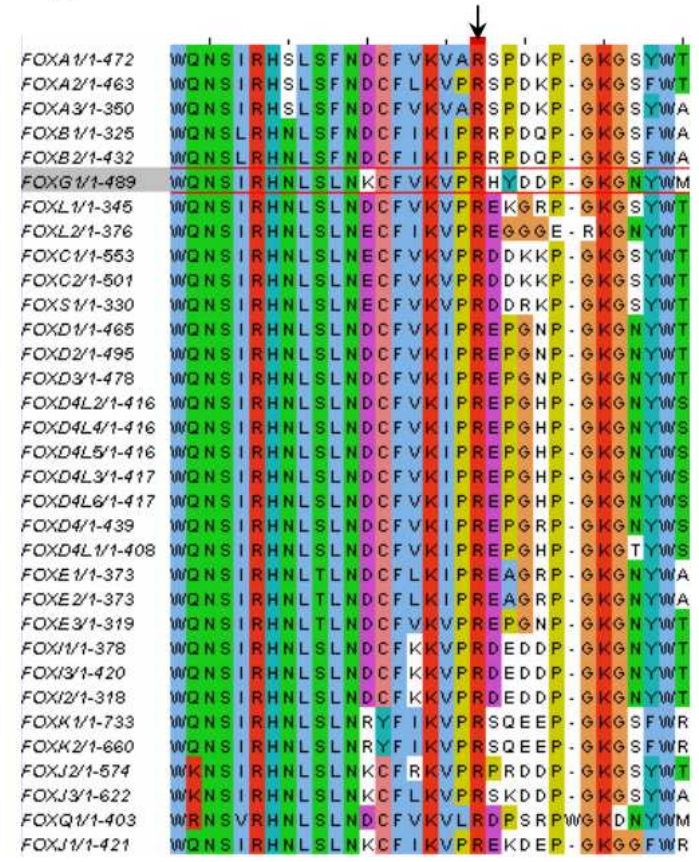

Figure 2. Arginine 244 (p.R244) is a conserved amino acid residue located within the forkhead domain of the FoxG1 protein. A) Schematic representation of the FoxG1 protein showing the poly-histidine tract (amino acids 47-57) and the relative position of the R244 amino acid. GBD: Groucho-binding domain; JBD: JARID-1B-binding domain; $\mathrm{N}$ and $\mathrm{C}$ : N- and C-termini, respectively. Alignment of the human FoxG1 protein sequence (ClustalW2: www.ebi.ac.uk/Tools/clustalw2/) in the close vicinity of the R244 residue with B) 15 multi-species orthologous and C) 33 human paralogous protein sequences imported from Ensembl Genome Browser v60 (www.ensembl.org/). Arginine 244 (R244) is indicated by an arrow.

\section{The p.R244C mutation affects the localisation of the FOXG1 protein}

To better understand the pathogenic effect of this mutation, we first investigated the subcellular distribution of the overexpressed FoxG1 mutant protein fused to GFP in HeLa and neuronal DAOY cells (Fig. 3). While the wild-type protein shows a homogeneous nuclear pattern excluding the nucleoli (Fig. 3A,B), the p.R244C mutant appears to aggregate as nuclear foci (Fig. 3E,F). The respective subcellular distribution of the FOXG1 mutant protein was confirmed by western-blot analysis of cell fraction protein extracts (data reviewed but not shown). Ricciardi and colleagues recently showed that the product of the CDKL5 gene, which is involved in the early onset seizure variant of Rett syndrome, localizes in nuclear speckles that are involved in the pre-mRNA processing in cells [Weaving et al. 2004; Ricciardi et al. 2009]. We then stained our cell models with an antibody directed against this structure (i.e. splicing factor SC-35) (Fig. 3C,G). We clearly observed that the FOXG1 mutant protein extensively, although not fully, localizes in nuclear speckles (Fig. 3H, white arrowheads), while the wild-type FOXG1 protein is more widely dispersed throughout the nucleus (Fig. 3D). This result suggests that the specific 
nuclear localisation of the p.R244C mutant in nuclear speckles may affect the functions of these nuclear domains, which are known to be involved in the assembly and/or modification of pre-mRNA splicing factors.

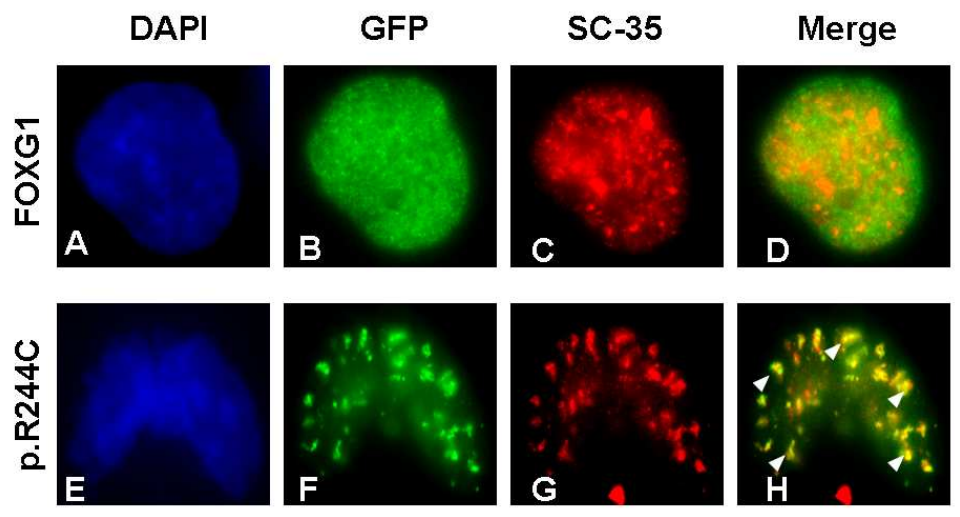

Figure 3. Immunofluorescence analysis of (HeLa/DAOY) cells transfected by pEGFP-FOXG1 (A-D) and pEGFPFOXG1_p.R244C (E-H). Fixed cells were mounted with Vectashield mounting medium with DAPI (A, E). Green fluorescence was observed by direct fluorescence (B, F) and SC-35 staining appears in red in immunolabeled cells (C, G). Merge images (green/red fluorescence) are shown (D, H).

\section{The p.R244C mutation affects the CDKN1A expression}

To investigate whether the p.R244C mutation located within the DNA-binding forkhead domain affects the expression of target genes, we studied expression of the cyclin-dependent kinase inhibitor (CKI) p21 CDKN1A gene in transfected wild-type FOXG1 or mutated FOXG1 neuronal SH-SY5Y cells. After transfection and collection of (GFP+)-transfected using a FACScan flow cytometer, total RNAs were extracted, converted to cDNA, and CDKN1A expression was studied by real-time RT-PCR. As reported in previous reports [Seoane et al. 2004; Kawauchi et al. 2009; Chan et al. 2009], we first observed that overexpression of the wild-type protein FOXG1 in cells results in a decrease ( 40\%) of CDKN1A expression as compared with untransfected cells (data not shown). Secondly, we showed that overexpression of the p.R244C mutant results in an increase (Fig. 4; $31 \pm 0.2 \%$ ) in $C D K N 1 A$ expression, suggesting that in contrast to the wild-type FOXG1 protein, this mutant loses its ability to repress $C D K N 1 A$ expression, at least partially, and that this misregulation may likely be due to its abnormal concentration in the nuclear speckles. 


\section{$10<$ First Author et al.>}

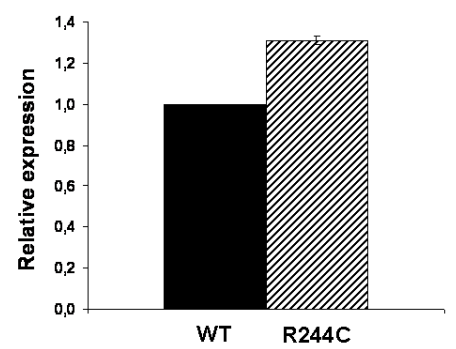

Figure 4. Real-time quantitation of CDKNIA mRNA levels in (GFP+)-wild-type (WT) and mutated (R244C) transfected cells Total RNA was converted to cDNA for quantitative real-time PCR with SYBR Green as the detection agent with the ABI Prism 7500 Sequence Detection System (Applied Biosystems). CDKNIA expression was studied by quantitative Reverse Transcriptase (RT)-PCR. RT-PCR was carried out in triplicate and expression levels were normalized to $H P R T$ as an endogenous control.

\section{DISCUSSION}

In this report, we describe two female patients with novel missense FOXG1 mutations. One of these variants, the p.P109L mutation, was also identified in her healthy father at the heterozygous state, suggesting that it is a nonpathogenic polymorphism. The other variant, the p.R244C mutation was not detected in more than 100 control female individuals of different ethnic origins, and FOXGl parent's screening and paternity testing using microsatellite markers suggest that the p.R244C mutation is a de novo mutation. This raises the total number of reported pathogenic missense mutations to three (p.F215L, p.P227K, and p.R244C). All of these mutations are located within the highly evolutionarily conserved DNA-binding forkhead domain of the protein (amino acids 181 to 275). However, up to now, their disruptive effect on the function of the FOXG1 protein has not been clearly shown. This novel mutation was identified in a girl with a combination of postnatal microcephaly, dyskinetic movement disorder with hand stereotypies, and relative preserved nonverbal communication. These clinical features are similar to those previously described in patients with the congenital variant of Rett syndrome [BahiBuisson et al. 2010]. Moreover, a simplified gyral pattern, as well as a delayed myelination, two features previously reported in FOXG1 mutation patients, are observed in our patient suggesting that these MRI abnormalities are key features to identify patients likely to carry FOXG1 mutations.

In our study, the overexpressed wild-type protein is localised throughout the nucleus excluding the nucleoli, as previously observed. Conversely, the p.R244C FoxG1 mutant aggregate as nuclear foci, and interestingly colocalizes with the splicing factor SC-35, a marker of the nuclear speckles. FoxG1 is a nuclear protein bearing a 
poly-histidine (His) repeat (11 His, amino acids 47-57) in its N-terminal part. As observed in our work, it has been shown that loss of DNA binding in other poly-Histidine(His)-containing nuclear proteins (such as the transcription factor MEOX2) [Salichs et al. 2009] with a point mutation is associated with their localization in intranuclear speckles. Recently, Ricciardi and colleagues showed that the product of the CDKL5 gene, which is involved in the early onset seizure variant of Rett syndrome, also localizes in nuclear speckles that are involved in the pre-mRNA processing in cells [Ricciardi et al. 2009]. Because nuclear speckles are thought to be the result of the aggregation of pre-mRNA splicing factors, including ribonucleoprotein particles (snRNPs) and arginin-serine-rich splicing factors, it has been suggested that any CDKL5 dysfunction results in an imbalance of the various components of the spliceosome machinery, which ultimately leads to alterations of the splicing pattern of a number of yet undefined RNA transcripts. Additional studies will be required to elucidate the functional consequences of the localization of mutant FOXG1 in nuclear speckles on the splicing machinery, and the possible link with CDKL5 in the pathological mechanisms of this disease.

Finally, it is well established that FoxG1 acts as a transcriptional repressor, with both direct and indirect mechanisms, in both DNA-binding dependent and independent manners. Target genes include cell cycle inhibitors, such as the cyclin-dependent kinase inhibitor 1A (p21, Cip1) (CDKN1A) gene [Seoane et al. 2004; Kawauchi et al. 2009; Chan et al. 2009]. FoxG1 is known to be a potent inhibitor of the transforming growth factor (TGF) $\beta$ regulated signalling by inhibiting the TGF $\beta$-dependent transcription of the CDKN1A gene, encoding a cell cycle protein that may induce cell cycle exit in neural precursors [Siegenthaler and Miller, 2005]. In this study, we showed that overexpression of FOXG1 in neuroblastoma cells reduces CDKNIA expression. This inhibitory role of FoxG1 on TGF $\beta$-induced $C D K N 1 A$ expression has already been described in earlier reports [Adesina et al. 2007; Chan et al. 2009]. The p.R244C FoxG1 mutant loses its effect, suggesting that this mutation affects the expression of CDKN1A at the transcriptional level in SH-SY5Y cells. During brain development, this mutant may induce cell cycle exit in neural precursors leading to severe microcephaly observed in mutated FOXG1 patient, in adult Foxg1 haploinsufficient mice [Shen et al., 2006], and in Fgf8 hypomorphic and null mice, which is likely due, at least in part, to alterations in Foxg1 expression [Storm et al., 2006].

In conclusion, we identified a novel missense mutation within the DNA-binding forkhead domain of FoxG1 in an 8-year-old girl, who presented the combination of postnatal microcephaly, dyskinetic movement disorders with hand stereotypies, relative good eye contact and frontal gyral simplification and myelination delay. The observation that the FOXG1 mutation associated with the disease state causes mislocalization of the protein in the nucleus offers an additional possible explanation for the loss of function of FoxG1 in mutated affected patients. This loss of function is associated with the loss of repressive activity against CDKN1A expression. Additional studies are required to elucidate the functional consequences of the nuclear speckle-localization of mutant FoxG1 proteins on the splicing machinery and its possible link with CDKL5 in the pathological mechanisms of this disease. 


\section{$12<$ First Author et al.>}

\section{REFERENCES}

Adesina AM, Nguyen Y, Mehta V, Takei H, Stangeby P, Crabtree S, Chintagumpala M, Gumerlock MK. 2007. FOXG1 dysregulation is a frequent event in medulloblastoma. J Neurooncol 85:111-1122.

Ariani F, Hayek G, Rondinella D, Artuso R, Mencarelli MA, Spanhol-Rosseto A, Pollazzon M, Buoni S, Spiga O, Ricciardi S, Meloni I, Longo I, Mari F, Broccoli V, Zappella M, Renieri A. 2008. FOXG1 is responsible for the congenital variant of Rett syndrome. Am J Hum Genet 83:89-93.

Bahi-Buisson N, Nectoux J, Girard B, Van Esch H, De Ravel T, Boddaert N, Plouin P, Rio M, Fichou Y, Chelly J, Bienvenu T. 2010. Revisiting the phenotype associated with FOXG1 mutations: two novel cases of congenital Rett variant. Neurogenetics 11:241-249.

Chan DW, Liu VW, To RM, Chiu PM, Lee WY, Yao KM, Cheung AN, Ngan HY. 2009. Overexpression of FOXG1 contributes to TGF-beta resistance through inhibition of p21WAF1/CIP1 expression in ovarian cancer. Br J Cancer 101:14331443 .

Kawauchi S, Kim J, Santos R, Wu HH, Lander AD, Calof AL. 2009. Foxg1 promotes olfactory neurogenesis by antagonizing Gdf11. Development. 2009 136:1453-1464.

Lee HH, Frasch M. 2004. Survey of forkhead domain encoding genes in the Drosophila genome: Classification and embryonic expression patterns. Dev Dyn 229:357-366.

Mencarelli MA, Spanhol-Rosseto A, Artuso R, Rondinella D, De Filippis R, Bahi-Buisson N, Nectoux J, Rubinsztajn R, Bienvenu T, Moncla A, Chabrol B, Villard L, Krumina Z, Armstrong J, Roche A, Pineda M, Gak E, Mari F, Ariani F, Renieri A. 2010. Novel FOXG1 mutations associated with the congenital variant of Rett syndrome. J Med Genet 47:49-53.

Murphy DB, Wiese S, Burfeind P, Schmundt D, Mattei MG, Schulz-Schaeffer W, Thies U. 1994. Human brain factor 1, a new member of the fork head gene family. Genomics 21:551-557.

Philippe C, Amsallem D, Francannet C, Lambert L, Saunier A, Verneau F, Jonveaux P. 2010. Phenotypic variability in Rett syndrome associated with FOXG1 mutations in females. J Med Genet. 47:59-65.

Regad T, Roth M, Bredenkamp N, Illing N, Papalopulu N. 2007. The neural progenitor-specifying activity of FoxG1 is antagonistically regulated by CKI and FGF. Nat Cell Biol 9:531-540.

Ricciardi S, Kilstrup-Nielsen C, Bienvenu T, Jacquette A, Landsberger N, Broccoli V. 2009. CDKL5 influences RNA splicing activity by its association to the nuclear speckle molecular machinery. Hum Mol Genet 18:4590-4602.

Rolando S. 1985. Rett syndrome: report of eight cases. Brain Dev 7:290-296.

John Wiley \& Sons, Inc. 
Salichs E, Ledda A, Mularoni L, Mar Alba M, De la Luna S. 2009. Genome-wide analysis of histidine repeats reveals their role in the localisation of human proteins to the nuclear speckles compartment. PLoS Genetics 5:1-12.

Seoane J, Le HV, Shen L, Anderson SA, Massagué J. 2004. Integration of Smad and forkhead pathways in the control of neuroepithelial and glioblastoma cell proliferation. Cell. 117:211-23.

Shen L, Nam HS, Song P, Moore H, Anderson SA. 2006. FoxG1 haploinsufficiency results in impaired neurogenesis in the postnatal hippocampus and contextual memory deficits. Hippocampus 16:875-890.

Siegenthaler JA, Miller MW. 2005. Transforming growth factor beta 1 promotes cell cycle exit through the cyclin-dependent kinase inhibitor p21 in the developing cerebral cortex. J Neurosci 25:8627-8636.

Solomon KS, Logsdon JM Jr, Fritz A. 2003a. Expression and phylogenetic analyses of three zebrafish FoxI class genes. Dev Dyn 228:301-307.

Solomon KS, Kudoh T, Dawid IB, Fritz A. 2003b. Zebrafish foxi1 mediates otic placode formation and jaw development. Development 130:929-940.

Storm EE, Garel S, Borello U, Hebert JM, Martinez S, McConnell SK, Martin GR, Rubenstein JL. 2006. Dose-dependent functions of Fgf8 in regulating telencephalic patterning centers. Development. 133:1831-1844.

Tao W, Lai E. 1992. Telencephalon-restricted expression of BF-1, a new member of the HNF-3/fork head gene family, in the developing rat brain. Neuron. 8:957-966.

Weaving LS, Christodoulou J, Williamson SL, Friend KL, McKenzie OL, Archer H, Evans J, Clarke A, Pelka GJ, Tam PP, Watson C, Lahooti H, Ellaway CJ, Bennetts B, Leonard H, Gécz J. 2004. Mutations of CDKL5 cause a severe neurodevelopmental disorder with infantile spasms and mental retardation. Am J Hum Genet 75:1079-1093. 